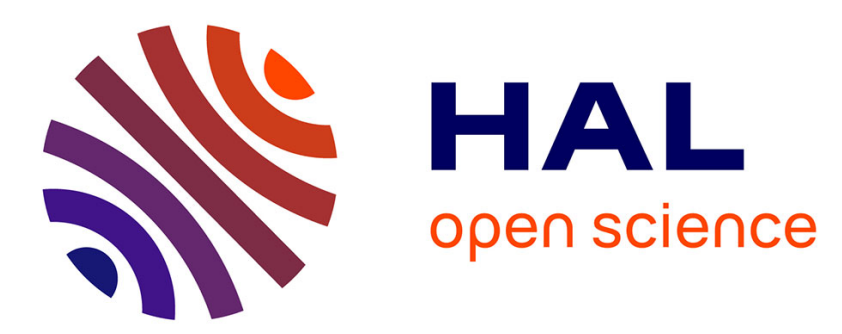

\title{
Alignment of Complex Fluids under Confinement and Flow
}

\author{
I. Koltover, S. Idziak, P. Davidson, Y. Li, C. Safinya, M. Ruths, S. Steinberg,
} J. Israelachvili

\section{> To cite this version:}

I. Koltover, S. Idziak, P. Davidson, Y. Li, C. Safinya, et al.. Alignment of Complex Fluids under Confinement and Flow. Journal de Physique II, 1996, 6 (6), pp.893-907. 10.1051/jp2:1996218 . jpa00248339

\section{HAL Id: jpa-00248339 https://hal.science/jpa-00248339}

Submitted on 1 Jan 1996

HAL is a multi-disciplinary open access archive for the deposit and dissemination of scientific research documents, whether they are published or not. The documents may come from teaching and research institutions in France or abroad, or from public or private research centers.
L'archive ouverte pluridisciplinaire HAL, est destinée au dépôt et à la diffusion de documents scientifiques de niveau recherche, publiés ou non, émanant des établissements d'enseignement et de recherche français ou étrangers, des laboratoires publics ou privés. 


\title{
Alignment of Complex Fluids under Confinement and Flow
}

I. Koltover $\left({ }^{1}\right)$, S.H.J. Idziak $\left({ }^{1}\right)$, P. Davidson $\left({ }^{1,3}\right)$, Y. Li $\left({ }^{1}\right)$, C.R. Safinya $\left({ }^{1, *}\right)$, M. Ruths $\left({ }^{2,4}\right)$, S. Steinberg $\left({ }^{2}\right)$ and J.N. Israelachvili $\left({ }^{2}\right)$

(1) Materials Department, Physics Department, and Biochemistry and Molecular Biology Program, University of California, Santa Barbara, CA 93106, USA

$\left({ }^{2}\right)$ Chemical Engineering and Materials Departments, University of California at Santa Barbara, Santa Barbara, CA 93106, USA

$\left({ }^{3}\right)$ Laboratoire de Physique des Solides $\left({ }^{* *}\right)$, Bât. 510, Université Paris Sud, 91405 Orsay Cedex, France

$\left({ }^{4}\right)$ Department of Chemistry, University of California at Santa Barbara, Santa Barbara, CA 93106, USA

(Received 2 December 1995, received in final form 29 January 1996, accepted 20 February 1996)

PACS.61.30.Eb - Experimental determinations of smectic, nematic, cholesteric, and other structures

PACS.64.70.Md - Transitions in liquid crystals

PACS.82.70.Kj - Emulsions and suspensions

\begin{abstract}
We report on a new application of the X-ray Surface Forces Apparatus [1-4] (XSFA) to the study of the alignment of complex fluid thin films under confinement and/or flow using synchrotron $\mathrm{X}$-ray scattering. The smectic A liquid crystal 8CB (4-cyano-4'-octylbiphenyl) and the hexagonal phases of a zwitterionic polyisoprene melt and of a lyotropic liquid crystal composed of sodium dodecyl sulfate (SDS), pentanol and water were investigated in the regime where the distances between the confining surfaces range from 0.4 to tens of microns. In this paper, we demonstrate that this new technique allows preparation of highly-oriented complex fluid samples suitable for high resolution $\mathrm{X}$-ray diffraction and scattering studies. In addition, we have directly measured forces across thin films of $8 \mathrm{CB}$ using the conventional Surface Forces Apparatus (SFA). The force measurements complement well the direct $\mathrm{X}$-ray imaging and provide indirect information on the sample alignment in films a few tens of Ångströms thick.
\end{abstract}

\section{Introduction}

The recently developed X-ray Surface Forces Apparatus (XSFA) [1] allows the study of complex fluid thin films under well-controlled experimental conditions of precisely measured confining gaps and variable shear flow. In particular, in-situ synchrotron $\mathrm{X}$-ray diffraction studies can be conducted on fluids in the "meso-scale" size regime between about $4000 \AA$ and a few microns. The first application of this technique to investigate the structure of a thermotropic smectic A liquid crystal under confinement and flow [1-4] revealed unexpected and interesting results. Well aligned domains of the smectic phase could be produced under confinement and were examined in-situ by X-ray scattering. Moreover, the behavior of the confined liquid crystal

$\left(^{*}\right)$ Author for correspondence (e-mail: safinya@engineering.ucsb.edu)

$\left(^{* *}\right)$ associé au CNRS

(C) Les Éditions de Physique 1996 
under low shear flow turned out to be very different from previously studied flow-induced behavior in the bulk samples [5], with effects of confinement dominating over the flow-induced alignment effects at small confinement gaps.

Confinement of complex fluid systems in thin films between two surfaces or in narrow pores is expected to strongly alter the collective structure of trapped molecules or molecular aggregates such as polymers, vesicles, biomembranes, or colloidal particles suspended in a liquid even in the absence of flow [6-11]. For example, molecular dynamics simulations [12] indicate that increasing static confinement may change the density, positional and orientational order of molecules and aggregates, especially as the decreasing gap size approaches an inherent length scale of the sample, such as the diameter of suspended colloidal particles or the radius of gyration of dissolved polymer coils and, ultimately, the size of the trapped solvent molecules themselves.

Alignment within complex fluid systems may also be induced dynamically, 1.e. by flow [5,13-15]. However, flow-induced structural effects do not require confinement and can occur in the bulk. An important difference between confinement-induced and flow-induced effects is that in the latter case the resulting structure is not at equilibrium. An understanding of the properties of complex fluid systems under flow conditions is of fundamental scientific interest and also of importance to many technological fields such as lubrication, the flow of colloidal and biological particles through narrow channels or biological pores. and the processing of ceramic and polymer composite materials and films.

The effects of confinement on molecular ordering and transport, as well as the phase behavior of liquids in small pores, have recently been studied with spectroscopic [10], calorimetric [11] and neutron scattering $[16,17]$ techniques. At the molecular level, the Surface Forces Apparatus (SFA) technique has allowed for direct force, friction, and rheological measurements of liquid films to be made [6-8]. However, until recently, complex fluids confined between two clean, smooth, well-characterized surfaces at precisely controllable separations have not been studied with a direct imaging probe such as X-ray or neutron diffraction. Such a probe should allow investigation of a number of important physical phenomena. First, by controlling the nature of the confining surfaces, it is possible [4] to separate the effects induced by surface-specific "epitaxial" interactions [9] from effects due to confinement; that is finite size effects. Second, by modifying the experimental apparatus so that the confining surfaces can also be moved with respect to each other, one can study the effects of shear on the fuid structure. Conceivably, by moving the surfaces fast enough and by decreasing the separation between the surfaces to a few Angströms one could achieve, simultaneously, extremely high shear rates and confinements.

Very interesting situations arise when the confinement and shear lead to a high degree of molecular alignment in a thin fluid sample, thus allowing detailed X-ray diffraction and scattering studies on these systems. Indeed, aligned samples are of crucial importance to perform structural studies of complex fluids since very valuable structural information is lost in the process of powder averaging and the signal-to-noise ratio of an aligned sample is always much larger than that of a powder. Moreover, aligned samples are extremely useful to study the anisotropic spectrum of thermal diffuse scattering (TDS) which gives access to the elastic moduli. The elastic moduli are obtained from the free energy; therefore, they give information on the thermodynamics of the phase. Though the smectic A phase of usual liquid crystals can often be aligned in bulk by application of a magnetic field for instance, other complex fluids such as viscous polymer melts or hexagonal liquid crystalline phases are notoriously difficult to align.

In this paper we describe the present state of the art of X-SFA technology developed since its first application [1], and report new results on the alignment of three different systems under confinement and shear flow. The classic rod-like liquid crystal 8CB (4-cyano-4'- octylbiphenyl) 
(a) smectic liquid crystal:

$8 \mathrm{CB}$ dimer

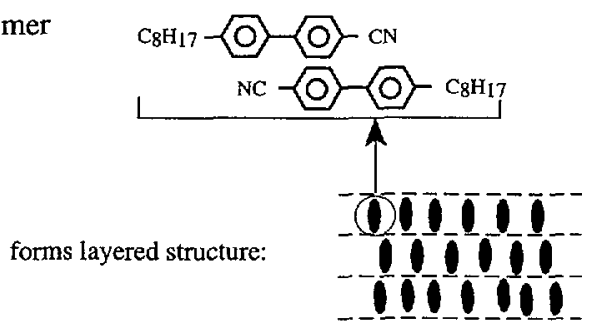

(b) Zwitterionic polyisoprene, MW=4650

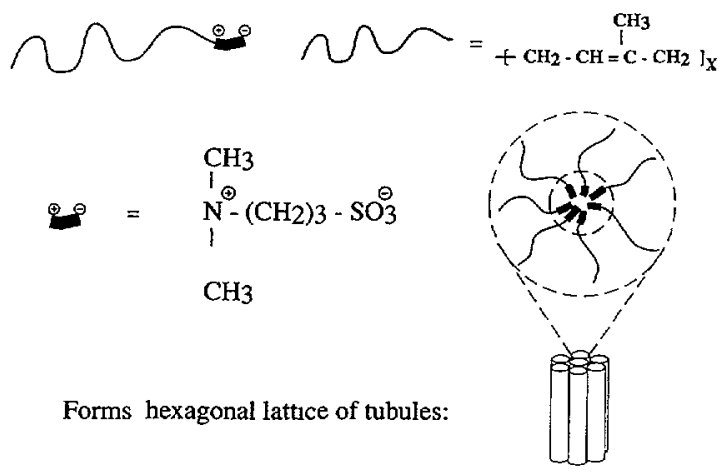

Fig. 1. - (a) 8CB forms a partially bilayered smectic A phase. (b) The zwitterionic polyisoprene forms a hexagonal phase.

has a smectic A-nematic phase transition at $32.5{ }^{\circ} \mathrm{C}$ [5]. Due to a permanent electrical dipole, the $8 \mathrm{CB}$ molecules tend to form antiparallel pairs giving rise to a partially bilayered smectic A phase (Fig. 1a) [18]. We have also studied the effects of confinement on a zwitterionic polyisoprene polymer melt $(\mathrm{MW}=4650)[19]$. The molecules of this polymer consist of a polar headgroup and a hydrocarbon tail (Fig. Ib). In this pure compound, the segregation between the headgroups and the tails induces the formation of tubes which assemble on a hexagonal lattice, giving rise to a hexagonal phase similar to those of lyotropic surfactant systems. The (SDS, pentanol, dodecane, water) system is a typical example of lyotropic surfactant system which has been extensively studied in the bulk, both under static $[20,21]$ and flow conditions $[15,22]$. A cut in its phase diagram (Fig. 2a) shows the existence of a hexagonal phase (Fig. $2 \mathrm{~b}$ ) in a wide range of concentration. For simplicity, the samples had no dodecane and had the composition: SDS $36.1 \mathrm{wt} . \%$, pentanol $7.78 \mathrm{wt} . \%$ and water $56.12 \mathrm{wt} . \%$. SDS and pentanol were purchased from Sigma and used without further purification.

\section{Experimental}

The XSFA is based on a conventional SFA Mk III [23], which was modified for simultaneous on-line use with an intense synchrotron X-ray beam passing normally through the sample and confining surfaces. In this device, the gap thickness between two atomically smooth surfaces can 
(a)

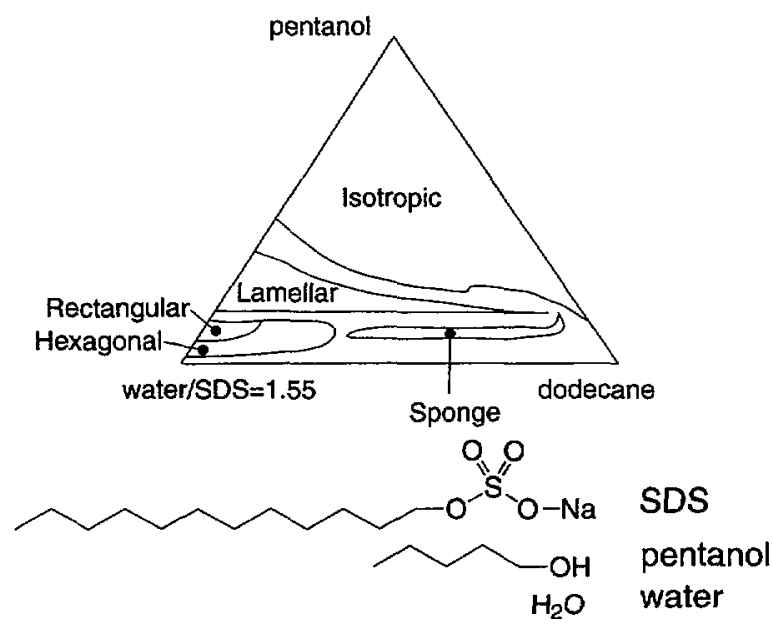

(b)

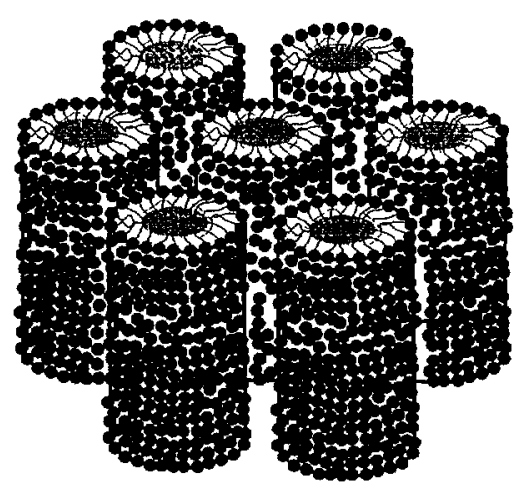

Fig. 2. - (a) Phase diagram of the (SDS, pentanol, dodecane, water) lyotropic system. (b) Schematic of the hexagonal phase showing the water separated tubes.

be continuously adjusted from a few $\AA$ ngströms to $>10 \mathrm{~mm}$, with control to $\pm 10 \AA$ by means of a three-stage coupled micrometer/differential micrometer/differential spring mechanism which was motorized for some of the experiments. The surfaces used are freshly-cleaved back-silvered mica sheets mounted in a crossed-cylinder geometry. The curvature allows the surfaces to be brought into single point contact, but also requires the use of very narrow $\mathrm{X}$-ray beams in order to image the volume of the sample close to the point of contact. For our experiments the apparatus is mounted on a stepper-motor driven $X Y Z$ translation stage installed on a Huber four-circle X-ray diffractometer (Fig. 3a). During the experiments the scattering volume of the sample is fixed at the center of rotation of the diffactometer.

The X-ray scattering measurements were conducted on the wiggler beamlines 6-2 and 10-2 at the Stanford Synchrotron Radiation Laboratory. A Si(111) double-bounce monochromator was used at $8 \mathrm{keV}$ with the beam focused at the sample position. A $125 \mu \mathrm{m}$ pinhole was placed several centimeters before the sample to provide proper collimation of the incident X-ray beam (Fig. 3b). The diffraction patterns were observed with a $180 \mathrm{~mm}$ MAR image-plate 2D $\mathrm{X}$-ray detector. The incoming resolution was determined by the slits on the diffractometer and 
(a)

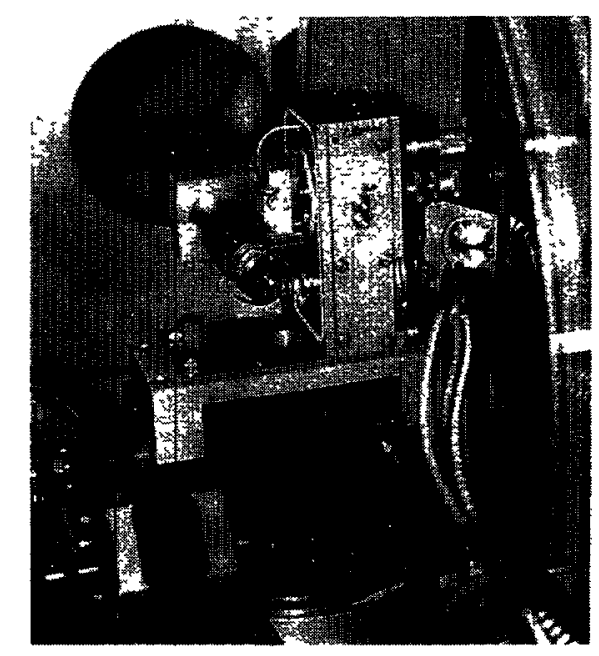

(b)

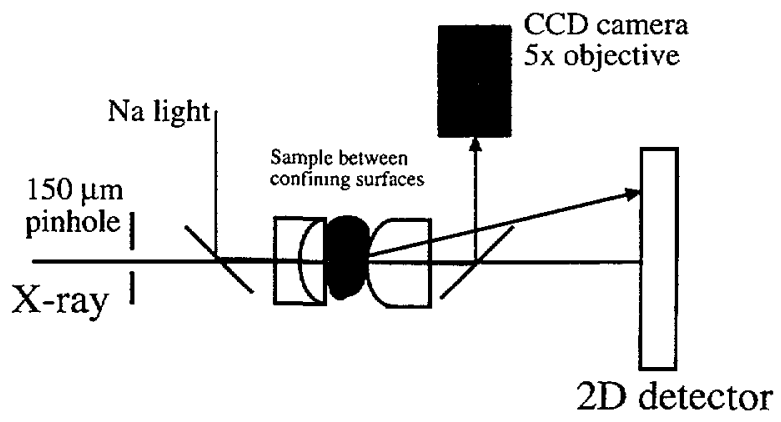

Fig. 3. - (a) A photograph of the X-ray Surface Forces Apparatus fitted onto the X-ray diffractometer. (b) Schematic of the experimental set-up showing the paths of the $\mathrm{X}$-rays and visible light in the system.

the outgoing resolution by the pixel size of the image-plate $(150 \mu \mathrm{m})$ and the distance from the sample to the detector $(400 \mathrm{~mm})$. In the experiments described here the resolution was $\delta q=0.001 \AA^{-1}$ where $q$ is the momentum transfer vector. Typical exposure times were about 20 minutes.

The gap between the two cylindrical surfaces was monitored simultaneously with the Xray measurements by observing the Newton's fringes created when the light from a sodium lamp was passed (coaxially with the X-ray beam) through the back-silvered mica surfaces and the confined sample. The Newton fringes interferometry allows the surface separations to be determined to within $500 \AA$. In order to monitor the fringes simultaneously with the X-ray measurements, we used a system of two $125 \mu \mathrm{m}$ thick aluminized mylar mirrors (Fig. 3b), which attenuated the X-ray beam by about $20 \%$ but did not contribute to the background scattering at the angles of interest for the systems studied. The Newton fringes were observed using a Nikon $5 \times$ long working distance objective and a CCD camera connected to a TV-monitor and video-recorder.

The cylindrical holders in our experiments were oriented so that the X-ray beam first passed through a surface with a vertically-oriented cylindrical axis, then through the sample and then 
through a horizontally-oriented surface (Fig. 3b). The latter was mounted on a spring attached to special piezoelectric bimorph sliding device [23], whereas the former was held fixed. This allowed us to apply a variable shear to our samples.

The confining surfaces used in these XSFA experiments must be transparent to X-rays and partially transparent to the visible sodium light to allow simultaneous $X$-ray access and optical gap thickness measurements. In order to satisfy these requirements we have developed two different kinds of surfaces (Fig. 4). The first type of surfaces, which we refer to as "the soft surfaces", were prepared by gluing a $5-6 \mu \mathrm{m}$ thick mica sheet on a cylindrical lens made of aluminum with a $45^{\circ}$ conical taper terminating at a $1 \mathrm{~mm}$ diameter hole drilled through the center of the lens (Fig. 4a). The mica was glued over the hole using a UV-cured optical glue. The second kind, referred to as "the hard surfaces", were prepared by first gluing a $4-8 \mu \mathrm{m}$ thick quartz sheet over the hole in the aluminum cylindrical-lens holder with a thermosetting epoxy and then gluing a $3-5 \mu \mathrm{m}$ thick mica sheet over the supporting quartz using the UVcured glue. In both the soft and the hard surfaces the cylindrical lenses had a $20 \mathrm{~mm}$ radius of curvature. Previous studies [4] have shown that the hard and soft surfaces behave in a different way as the latter ones tend to deform to accommodate the viscoelasticity of the sample.

The $8 \mathrm{CB}$ and zwitterionic polyisoprene experiments were conducted in open air and room temperature. The studies of lyotropic materials were also performed at room temperature but in a sealed environment. Samples were loaded by carefully placing a drop between the two surfaces after the XSFA had been installed and aligned. A small reservoir containing the same sample was also enclosed in the sample chamber in order to establish vapor pressure equilibrium for these water-rich lyotropic materials.

\section{Results and Discussion}

\subsection{Alignment of the Smectic A Phase of 8CB}

3.1.1. Force Measurements. - On the extremely hydrophilic freshly-cleaved mica surfaces used in our experiments, $8 \mathrm{CB}$ pairs are known to orient with their long axes parallel to the surfaces [24] (planar orientation). In order to check this epitaxial orientation of the $8 \mathrm{CB}$ molecules near the mica surfaces we have performed force-measurements with a conventional MkIII Surface Forces Apparatus [23]. Importantly, these force measurements yield information on the structure of the confined films in the gap regime of a few tens of Angströms, which was not imaged directly with the X-ray technique. Forces were measured between conventional SFA rigid mica surfaces of $20 \mathrm{~mm}$ radius of curvature. $8 \mathrm{CB}$ films were studied in both the nematic $\left(35^{\circ} \mathrm{C}\right)$ and smectic $\left(25^{\circ} \mathrm{C}\right)$ phases. The results of the force measurements are shown in Figure 5. Across the nematic film (Fig. $5 \mathrm{a}$ ) at separations below about $60 \AA$ the force is oscillatory as a function of the film thickness with a mean periodicity of $9-10 \AA$, which is about the same as the mean diameter of the $8 \mathrm{CB}$ antiparallel pairs [18]. These oscillations correspond to the squeezing-out of successive layers of $8 \mathrm{CB}$ molecules aligned in the planar orientation. The increasing amplitude of the oscillations with decreasing gap thickness indicates that the oriented pairs have also become positionally ordered into layers parallel to the confining surfaces within about $20-30 \AA$ of the surfaces [6,25] (Fig. 5a inset). The last two layers of molecules of $17 \AA$ thickness could not be removed even with a very large applied compressive pressure. This last layer probably consists of a monolayer of $8 \mathrm{CB}$ molecules strongly, perhaps epitaxially bound to each surface. Across the smectic film (Fig. 5b) strong long-range riscoelastic forces made it difficult to accurately measure the equilibrium force-curves. The data shown correspond to the gap sizes where the forces start to deviate from the background long-range force, which defines the "zero" force in Figure 5a. The additional force of approximately $50 \mathrm{~m} \mathrm{~N} / \mathrm{m}$ arising from the 
(a)

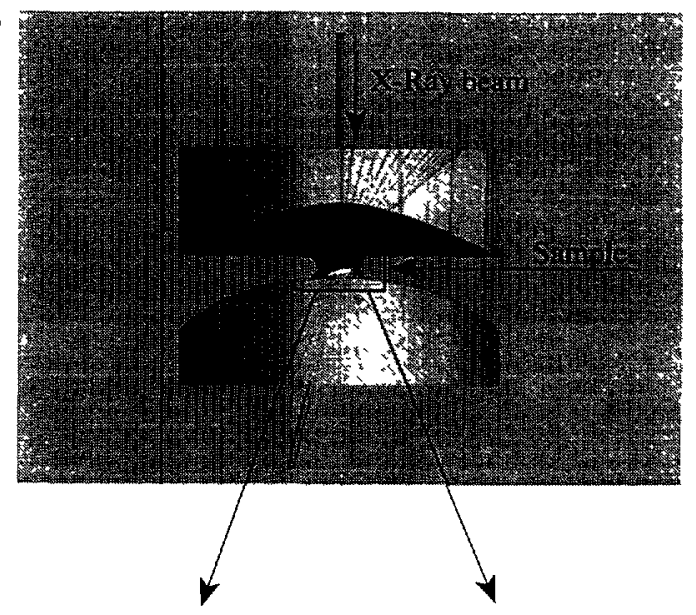

(b)

Soft surface

(c) Hard surface

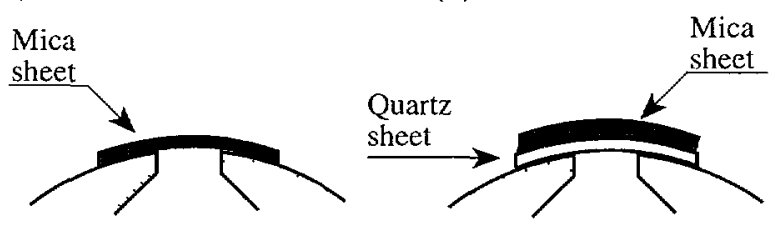

(d) Solid surface

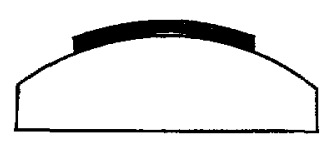

Fig. 4. - (a) Geometry of the surfaces in an X-SFA experiment showing the X-rays scattered by a sample confined between two cylindrical surfaces (disk mounts). (b) "soft surfaces" (c) "hard surfaces" (d) "conventional SFA surfaces".

high viscosity of the smectic sample makes an accurate determination of the magnitude of the force in the smectic phase very difficult. One can clearly see, however, that the smectic force curve has distinct kinks at the gap sizes equal to the diameter of the $8 \mathrm{CB}$ pairs, indicating that the pairs are again oriented parallel to the surfaces. The inset of Figure 5b also shows the same forces as measured across smectic film between two "hard" surfaces (Fig. 5b) used in the XSFA. The force curve exhibits the same features as that measured between the completely rigid surfaces with the conventional SFA. However, in this case, the slight elastic compliance of the surfaces causes them to flatten at these very small gaps, thereby magnifying the interaction area and, consequently, the measured force. Thus, in this case we were not able to remove the last $25 \AA$ of sample between the surfaces, corresponding to the last three layers of $8 \mathrm{CB}$ pairs. The force measurements, however, do not reveal whether the pairs are also positionally ordered within each of these quasi-layers. Traditional SFA measurements, which can be used to determine the structure along the normal to the surfaces, and XSFA measurements, which determine the structure in the plane of the surfaces, complement each other and can give a very complete description of the behaviour under confinement. 

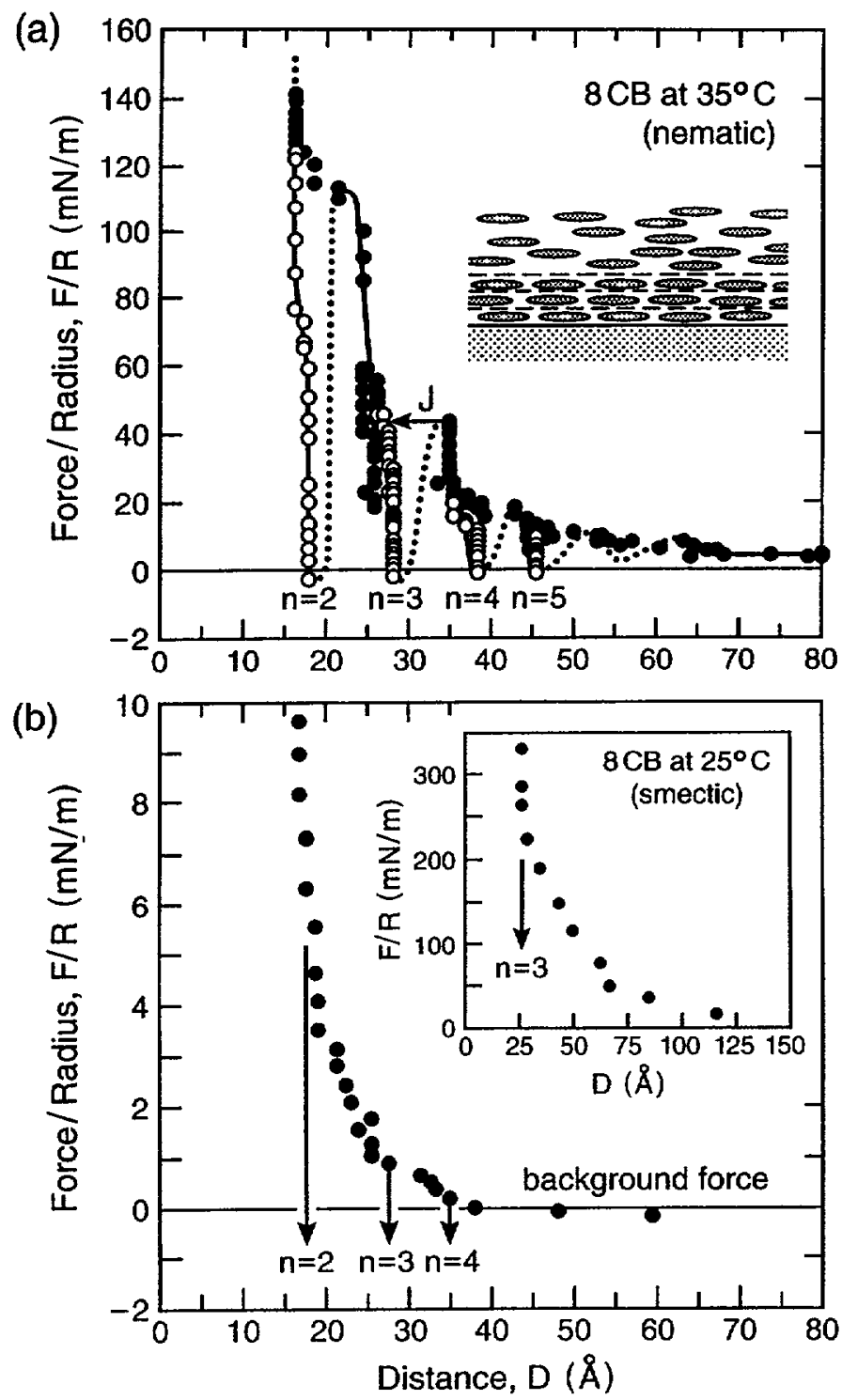

Fig. 5. - (a) Measured force between two SFA surfaces across $8 \mathrm{CB}$ in the nematic phase $\left(35^{\circ} \mathrm{C}\right)$ in the planar orientation. Filled circles: forces measured on approach or compression; open circles: on separation. (b) Measured force across a planarly oriented film of $8 \mathrm{CB}$ in the smectic phase $\left(25^{\circ} \mathrm{C}\right)$ on compression. The arrows indicate kinks in the repulsive force occurring at separations that correspond to discrete layers of thickness $\approx 10 \AA$ as measured in the nematic phase. The inset shows a similar compression of material between the same "hard surfaces" as used in the X-SFA.

3.1.2. X-ray Measurements. - From the previous section, we know that the smectic layers are oriented perpendicular to the confining surfaces, allowing us to see the diffraction peaks of an aligned sample in our experimental geometry. Figure 6a shows a typical 2D image 

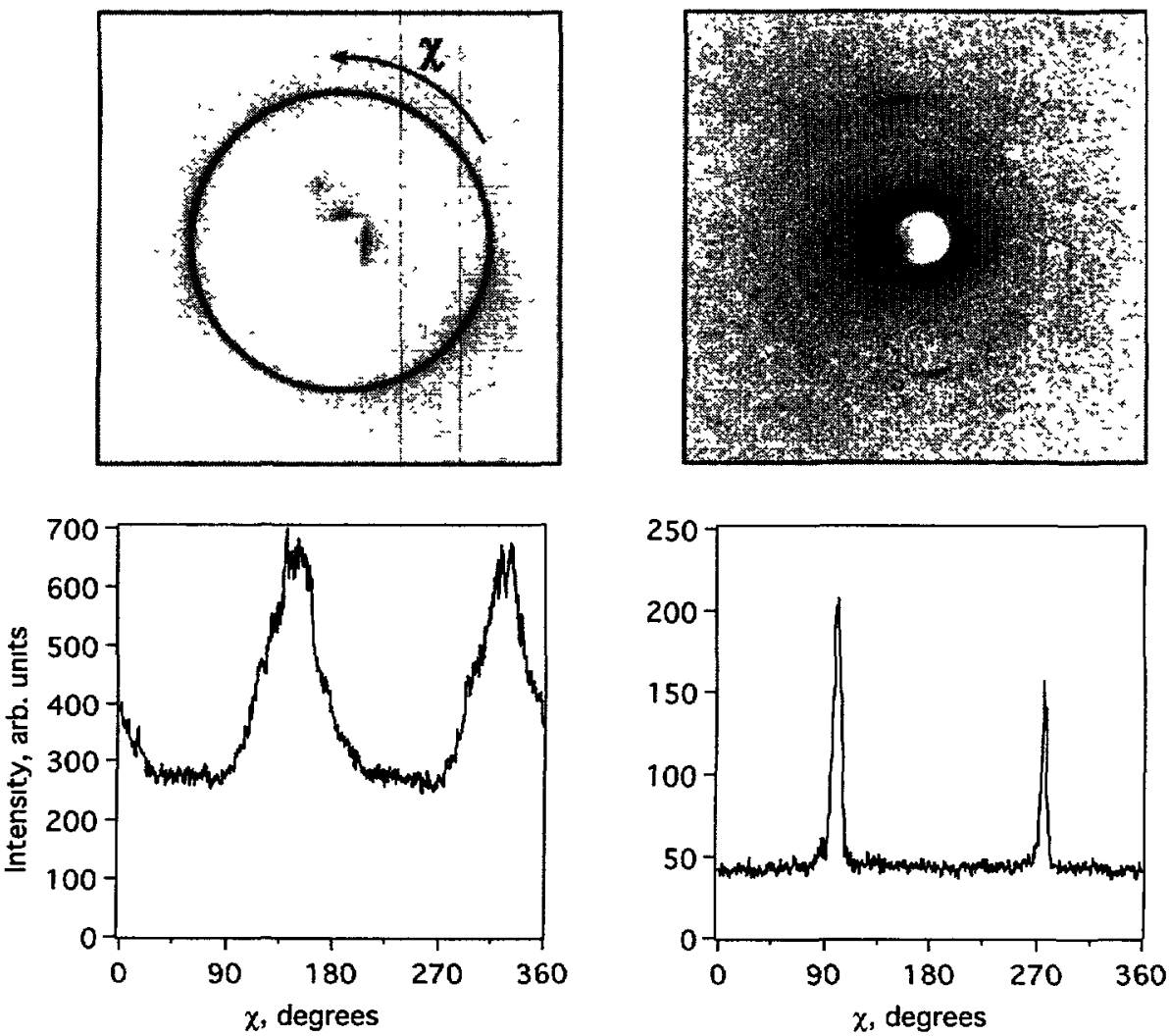

a)

b)

Fig. 6. - X-ray diffraction patterns and $\chi$-scans of $8 \mathrm{CB}(\mathrm{a})$ in bulk (b) confined in a $4 \mu \mathrm{m}$ wide gap.

of a diffraction pattern from a very thick film of $8 \mathrm{CB}$ in the smectic $A$ phase, held in the $\mathrm{X}-\mathrm{SFA}$. The sharp ring at the center is the characteristic diffraction peak of $8 \mathrm{CB}$ arising from the smectic structure. By taking appropriate cuts through the points of the $2 \mathrm{D}$ diffraction pattern, one can extract information corresponding to the various $1 \mathrm{D} X$-ray scans obtainable with a conventional point detector. For example, by taking the scattering intensities of points lying on a circle (centered on the incident X-ray beam) in the direction of the $\chi$ arrow in Figure $6 \mathrm{a}$, one obtains a conventional mosaic 1D $\chi$-scan. In general, a powder sample, consisting of many small randomly oriented domains, would appear as a flat line in this scan. A partially ordered sample would produce broad peaks, where the peak widths would indicate the degree of orientation of the domains present. Finally, an extremely ordered sample would manifest itself by producing very sharp peaks whose angular positions on the $\chi$-scan would reveal the preferred directions of the sample symmetry axes. In the case of $8 \mathrm{CB}$, the peak positions simply indicate the orientation of the normal to the smectic layers. Thus the very broad angular extent of the peaks in Figure 6a indicates that the sample consists of many smectic domains which are very slightly oriented with respect to the cylindrical confining surfaces. The partial orientation observed in Figure 6 a most likely results from the influence of the flow which took place in the sample during filling. The mosaic of this sample as determined from the 

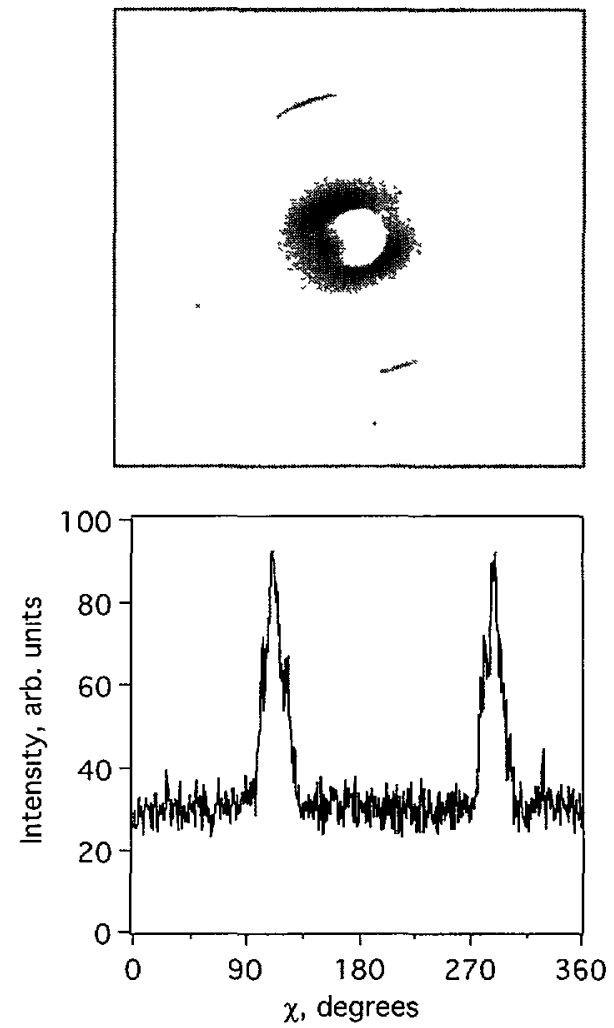

a)
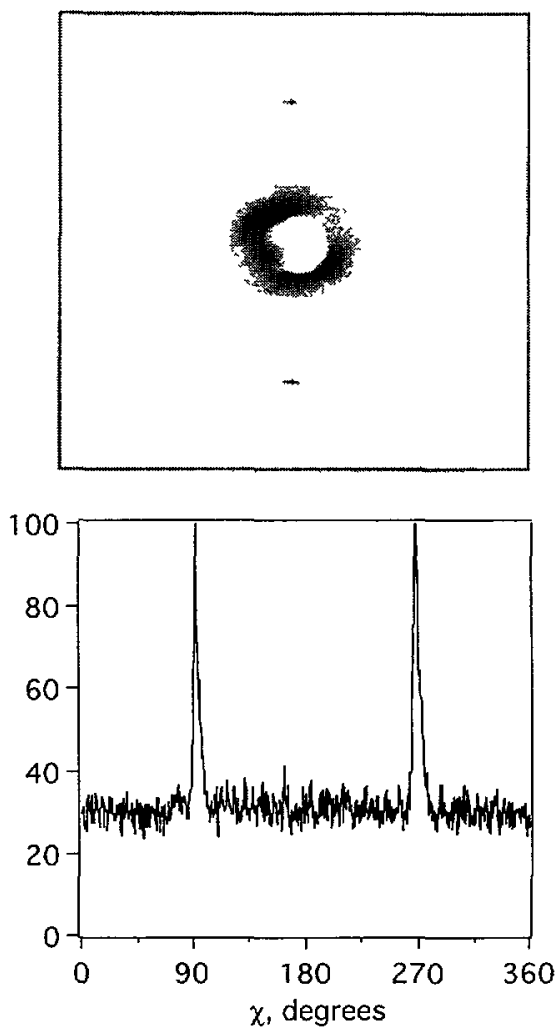

b)

Fig. 7. - X-ray diffraction patterns and $\chi$-scans of $8 \mathrm{CB}$ (a) at rest (b) under a shear rate of $150 \mathrm{~s}^{-1}$ The exposure time for (b) was 5 minutes, while it was 20 minutes in (a)

$\lambda$-scan is as high as 90 degrees (FWHM). In contrast, it is important to emphasize the high degree of alignment achieved with the $8 \mathrm{CB}$ sample confined between "hard surfaces" at a few microns gap size, as shown in Figure $6 \mathrm{~b}$. The mosaic of the sample in this case is only about 5 degrees (FWHM) which is comparable to the high alignments achieved in some cases by application of a magnetic field. This level of alignment is easily obtained and reproducible. It is also important to note that this very high degree of orientation is achieved for a relatively thick $(4 \mu \mathrm{m})$ sample, giving a relatively high X-ray scattering signal. This is clearly advantageous compared to some other techniques capable of achieving high degrees of alignment, such as in free-standing films or ultra-thin films on substrates. Moreover, if the surfaces are very carefully prepared, significantly better level of alignments may exceptionally be obtained with mosaicities of about 3.5 degrees. The use of "soft surfaces" [4] also helps to reach even lower mosaicities of 2 degrees. This is due to the fact that "soft surfaces" can flatten to better accommodate domain coarsening.

The state of alignment of a confined 8CB film can be further improved by shearing it between hard surfaces. Figure 7 shows the X-ray images and corresponding $\chi$-scans of the smectic phase of $8 \mathrm{CB}$ between hard surfaces at $1 \mu \mathrm{m}$ separation both at rest and under shear. The alignment of the sample clearly improves when a shear rate of about $150 \mathrm{~s}^{-1}$ is applied. The mosaicity 
of the sample decreases to less than 2 degrees (FWHM). Note also that the exposure time for the sample under shear ( 5 minutes) was 4 times less than that for the sample with no shear applied. Once aligned this way, the sample remains stable for hours.

In general, the flow-induced alignment may promote or compete with the alignment induced by the nature of the confining surfaces, thus leading to different orientations in the sample for different confining gaps or shear rates. It is thus interesting to note that the overall direction of the alignment changes under the applied shear. The peaks in Figure $7 \mathrm{~b}$ are centered around $90^{\circ}$ and $270^{\circ}$, indicating that the smectic layers in this case become aligned along the direction of shear flow in the sample. This alignment agrees with the orientation diagram previously observed [5] for bulk samples under shear flow conditions.

\subsection{Alignment of Hexagonal Phases}

3.2.1. Zwitterionic Polymer. - We have also applied the X-SFA technique to study the confinement induced alignment of a zwitterionic polyisoprene polymer melt in its hexagonal phase. Figure 8 shows two $\mathrm{X}$-ray scattering patterns and corresponding $\chi^{- \text {-scans }}$ of this system. The image in Figure $8 \mathbf{a}$ is from a thick sample $(d \approx 1 \mathrm{~mm})$. It only shows the $\left(\begin{array}{ll}1 & 0\end{array}\right)$ diffraction ring
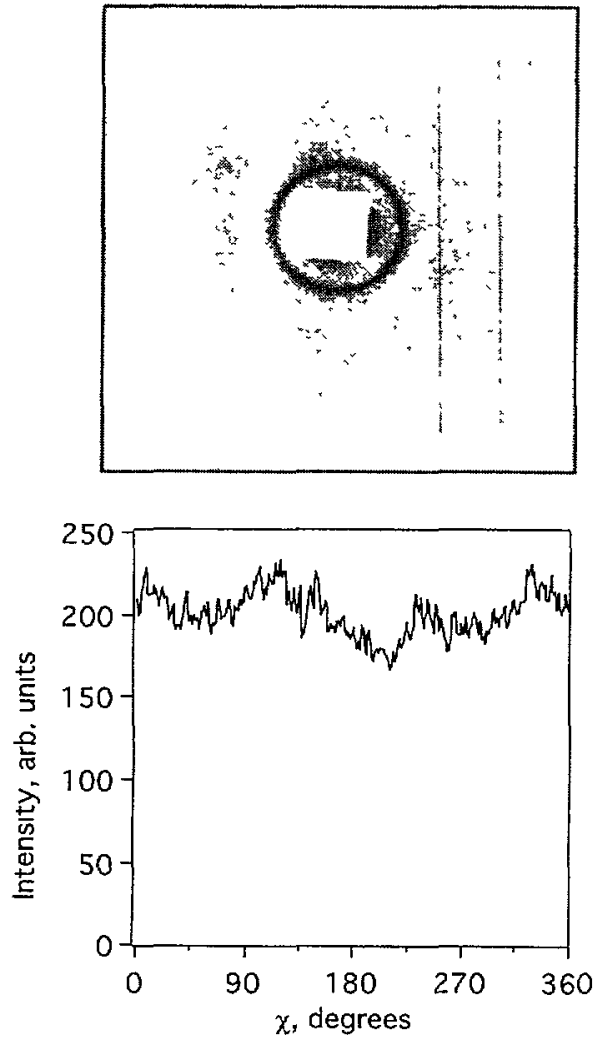

a)
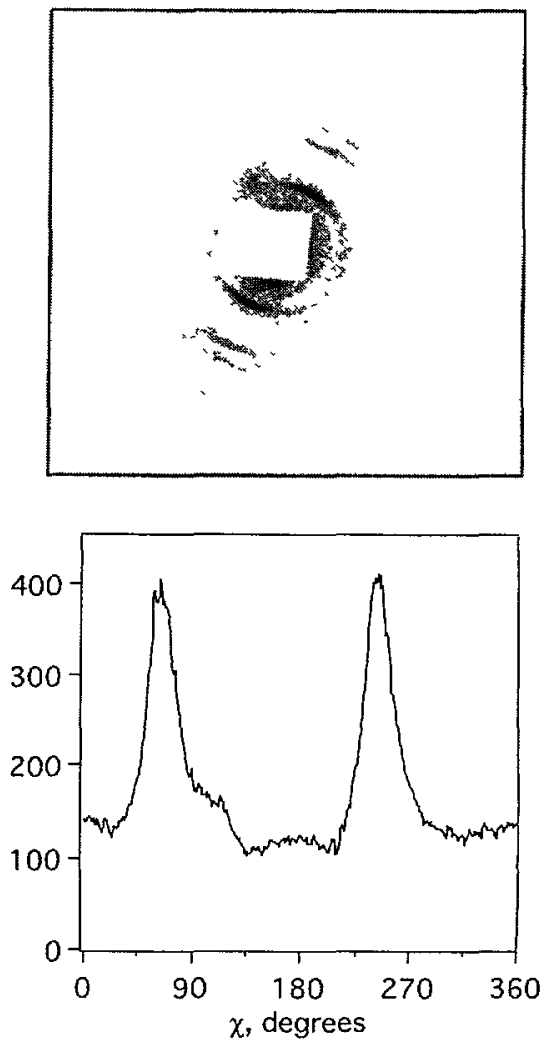

b)

Fig. 8. - X-ray diffraction patterns and $\chi$-scans of the zwitterionic polyisoprene (a) in bulk (b) confined in a several $\mu \mathrm{m}$ wide gap. 
of a powder (randomly oriented) sample. The image in Figure $8 \mathrm{~b}$ is from a sample confined within a gap size of several microns between two soft surfaces. One can see the $\left(\begin{array}{ll}1 & 0\end{array}\right),\left(\begin{array}{ll}1 & 1\end{array}\right)$ and $\left(\begin{array}{ll}2 & 0\end{array}\right)$ peaks. The sample is clearly in a well aligned state. The arrangement of the peaks on the $2 \mathrm{D}$ image suggests that the tubules are oriented along the mica surfaces with their axis in a direction almost exactly at a $45^{\circ}$ angle to the directions of the two cylindrical axes of the confining surfaces. The sample mosaic spread is about $30^{\circ}$ as determined from the corresponding $\chi$-scans. This alignment in a preliminary experiment is just slightly less than the one obtained by shear-alignment on a similar viscous polymer system (approximately 25\%) [26]. This is one of few experiments where one can achieve alignment in a highly viscous ordered polymer systems for X-ray diffraction studies.

3.2.2. Lyotropic Surfactant System. - Figure 9 shows two X-ray scattering patterns and corresponding $\chi$-scans of the (SDS, pentanol, water) system in its hexagonal phase. The image in Figure $9 \mathrm{a}$ is from a bulk sample (gap size $\approx 1 \mathrm{~mm}$ ) and displays the isotropic $\left(\begin{array}{ll}1 & 0\end{array}\right)$, and $\left(\begin{array}{ll}1 & 1\end{array}\right)$ scattering rings typical of a powder sample of hexagonal phase. The $\chi$-scan shows that this powder is a coarse one with large domains randomly oriented with respect to the cell geometry. In contrast, reducing the gap size to a few tens of microns yields highly aligned domains giving rise to scattering patterns of a very striking 6-fold symmetry (Fig. 9b). The differences in the

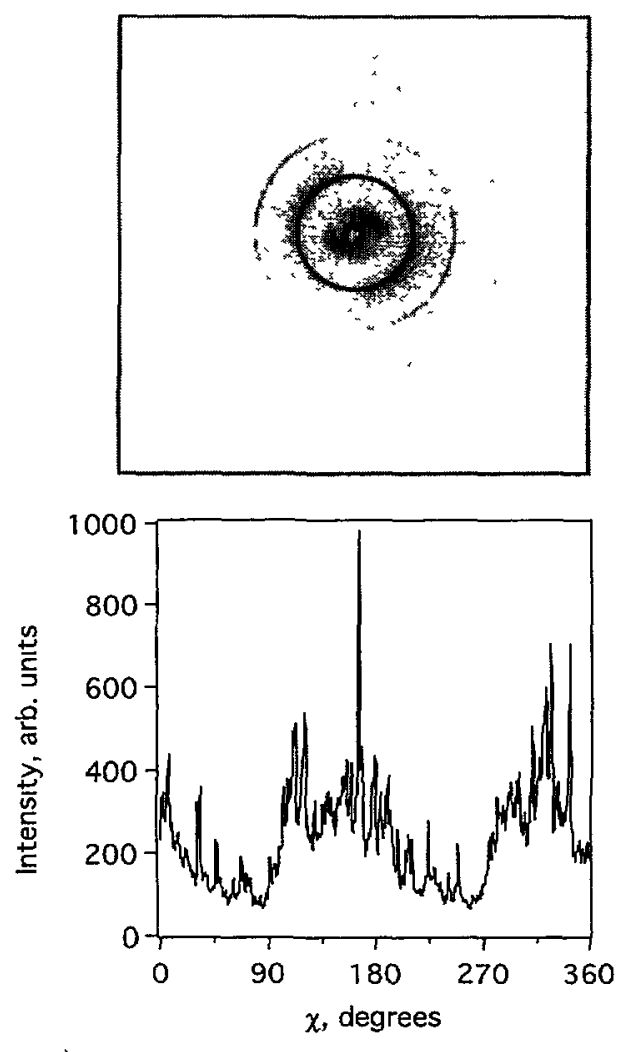

a)
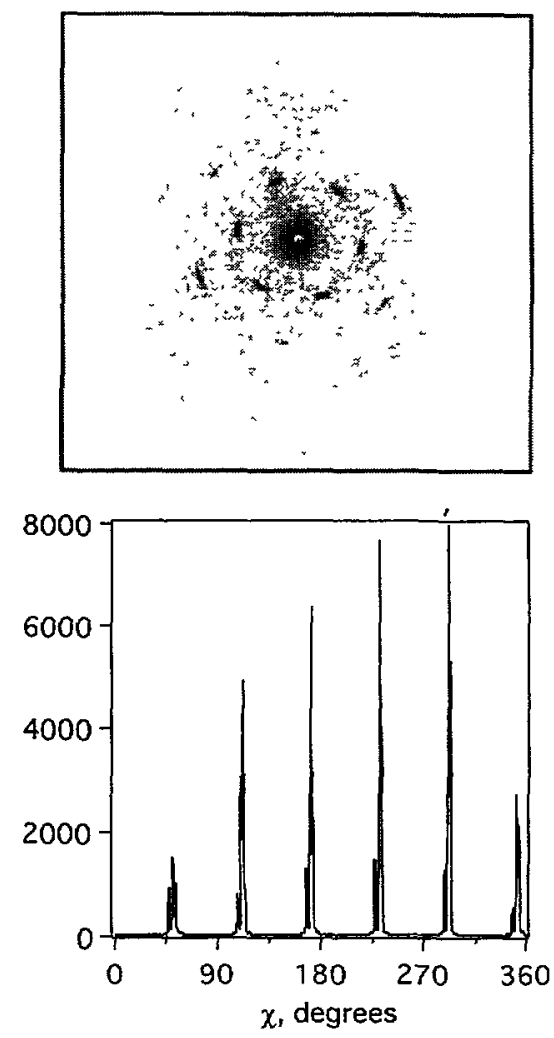

b)

Fig. 9. - X-ray diffraction patterns and $\chi$-scans of the lyotropic hexagonal phase (a) in bulk (b) confined in a $30 \mu \mathrm{m}$ wide gap. 
hexagonal reflection intensities simply result from a small misalignment of the surfaces with respect to the X-ray beam. Close inspection of the $\chi$-scan, in a range of 5 degrees around the Bragg reflections, shows that there are actually three unequal groups of domains in the beam, the main one having a mosaicity of less than 2 degrees (FWHM). Although there have been numerous structural studies of hexagonal phases of complex fluids, Figure $9 \mathrm{~b}$ is one of the few which shows such a high level of alignment. This outstanding domain growth was achieved in minutes by simply placing the sample between the two surfaces, squeezing the surfaces into contact, and then separating the surfaces approximately $30 \mu \mathrm{m}$ over 30 minutes. In contrast to the zwitterionic polymer system, the surfactant tubes are anchored perpendicularly to the surfaces. In the absence of extensive studies of the anchoring of hexagonal phases on mica substrates, it is difficult to understand this difference between the two systems.

The interest of using aligned samples compared to powders is illustrated by the signal-tonoise ratios of the $\left(\begin{array}{ll}1 & 0\end{array}\right)$ reflection in 10 minutes exposures which jumps from 20 for the bulk sample to more than 400 for the confined one. Such high signal-to-noise ratios allows detection of the $\left(\begin{array}{ll}2 & 0\end{array}\right)$ and $\left(\begin{array}{ll}2 & 1\end{array}\right)$ reflections on close inspection of the scattering pattern which was impossible for powders. This alignment procedure is similar to the strand drawing (pin and cup) technique $[27,28]$, but it allows direct X-ray access to the $2 d$ lattice which is forbidden by the pin and cup method. Furthermore, the high degree of alignment displayed by this confined hexagonal phase in principle also allows study of the X-ray diffuse scattering. This important kind of study [29] gives access to the elastic moduli of the phase which take part of the free energy.

Such a well aligned sample of hexagonal phase breaks into a coarse powder if it is quickly squeezed down to a few $\mu \mathrm{m}$. However, the alignment is retained if the squeezing takes place slowly at typical rates of $5 \mu \mathrm{m}$ per hour. Squeezing the sample must involve molecular diffusion, a process which has its own diffusion constant. If the stress is applied at a rate larger than the single domain can stand, then other processes will appear involving the creation of structural defects resulting in the breaking up of the aligned sample.

\section{Conclusion}

The results described above about very different systems show that the XSFA technique is a versatile new tool and probe for investigating the structure of complex fluids aligned by confinement and/or shear. Thus, the XSFA appears as a very valuable technique to grow single domains of complex fluids for in-situ diffraction and thermal diffuse scattering (TDS) experiments in systems otherwise difficult to align. These TDS experiments are of crucial importance since they allow us to measure the elastic moduli or their combinations. Since these moduli derive directly from the intermolecular interactions, TDS is an efficient tool to discriminate between various models describing interactions and self-assembling forces. TDS is a much stronger phenomenon in complex fluids than in crystals. In addition to the fact that fluctuations are intrinsically stronger in systems of low dimensionality, this also arises from the large size of the unit cell of complex fluids, usually about two orders of magnitude larger than crystals. Since the ratio of the intensities of the Bragg to TDS components is proportional to $N$, the number of unit cells in the scattering volume [30], the relative intensity of the TDS component is amplified by 4 to 6 orders of magnitude in systems with very large lattice spacings. The interest of using aligned samples for this kind of study can be illustrated by considering the lyotropic fluid lamellar $L_{\alpha}$ phase. The first studies $[20,21]$ performed on powders yielded accurate values of only one elastic modulus whereas by using techniques developed for orienting lyotropic lamellar phases [31,32], more accurate determination [33] of both moduli and in turn the complete free energy are feasible. These aligned samples had relatively poor 
mosaics ranging from 2 to 10 degrees. In contrast, the XSFA produces much better aligned samples. The same study of the lyotropic hexagonal phase is presently under way.

Moreover, the single domains prepared in the XSFA can be further manipulated by applying a shear as well as a uniaxial compression or dilation. The awaited availability of extremely high brilliance $\mathrm{X}$-ray beams with cross-sectional areas of order of a few $\mathrm{mm}$ will make possible the study of these single domains in rheological conditions by time resolved $\mathrm{X}$-ray scattering and therefore the obtention of a deep understanding of their mechanical properties. From this point of view, it will be also important and exciting to combine in future experiments the X-ray imaging with simultaneous on-line force measurements.

\section{Acknowledgments}

We gratefully acknowledge conversations with P. Pincus. C.R.S. and J.N.I. gratefully acknowledge partial support by the Office of Naval Research under grant No0014-93-1-0269. C.R.S. gratefully acknowledges partial support from the Los Alamos-UC Campus Collaborative Research Initiative (LACOR) under award UC-94-8-A-221. P.D. gratefully acknowledges support from a NATO research fellowship. M.R. thanks the Neste Foundation for financial support. The synchrotron X-ray scattering experiments were carried out at beamlines 6-2 and 10-2 at the Stanford Synchrotron Radiation Laboratory, which is supported by the U.S. Department of Energy. The Materials Research Laboratory at Santa Barbara is supported by NSF under grant No. DMR-9123048.

\section{References}

[1] Idziak S. H. J., Safinya C. R., Hill R. S., Kraiser K. E., Ruths M.. Warriner H. E., Steinberg S., Liang K. S, and Israelachvili J. N., Science 264 (1994) 1915.

[2] Idziak S. H. J., Safinya C. R., Sirota E. B., Bruinsma R. F., Liang K. S. and Israelachvili J. N., in "Structure and Flow in Surfactant Solutions", C. A. Herb and R. K. Prud homme Eds. (American Chemical Society. Washington. DC, 1994).

[3] Idziak S. H. J., Koltover I., Liang K. S. , Israelachvili J. N. and Safinya C. R. , Int. J. Thermophys. 16 (1995) 299.

[4] Idziak S.H.J., Koltover I., Israelachvili J.N. and Safinya C.R., Phys. Rev. Lett. 76 (1996) 1477.

[5] Safinya C. R., Sirota E. B.. Plano R. and Bruinsma R. F., J. Phys. Condens. Matt. 2 (1990) SA365; Safinya C. R., Sirota E. B. and Plano R., Phys. Rev. Lett. 66 (1991) 1986.

[6] Isrealachvili J. N., "Intermolecular and Surface Forces" (Academic Press. London \& New York, 1985 (1st edition), 1991 (2nd edition)).

[7] Van Alsten J. and Granick S., Phys. Rev. Lett. 61 (1988) 2570.

[8] Klein J.. Perahia D. and Warburg S., Nature 352 (1991) 143; Israelachvili J. N.. Homola A. M. and McGuiggan P. M., Scrence 240 (1988) 189.

[9] Als-Nielsen J., "Topics in Current Physics", W. Schommers and P. V. Blackenhagen, Eds. (Springer-Verlag, Verlin 1987) p. 181; Pershan P. S., J. Phys. Colloq. France 50 (1989) C7-1 ; Jerome B., Rep. Prog. Phys. 54 (1991) 391 and references therein.

[10] See e.g. Drake J. M. and Klafter J., Phys. Today 43 (1990) 46 and references therein; Drake J. M.. Klafter J. and Levitz P.. Science 251 (1991) 1574.

[11] Bellini T. et al., Phys. Rev. Lett. 69 (1992) 788. 
[12] Schoen M., Diestler D. J. and Cushman J. H., J. Chem. Phys. 87 (1987) 5464; Rhykerd C. L. Jr., Schoen M., Diester D. J. and Cushman J. H., Nature 330 (1987) 461; Thompson P. A., Robbins M. O. and Grest G. S., Phys. Rev. Lett. 68 (1992) 3448.

[13] Weissenberg K., Nature 159 (1947) 310; Bruinsma R. F. and Safinya C. R., Phys. Rev. A 43 (1991) 5377.

[14] Clark N. A. and Ackerson B. J., Phys. Rev. Lett. 44 (1980) 1005.

[15] Safinya C. R., Sirota E. B.. Bruinsma R. F., Jeppesen C., Plano R. J. and Wenzel L. J., Science 261 (1993) 588.

[16] Lin M.Y., Sinha S.K., Drake J.M., Wu X.-I., Thiyagarajan P. and Stanley H.B., Phys. Rev. Lett. 72 (1994) 2207.

[17] Lambooy P., Russell T.P., Kellogg G.J., Mayes A.M., Gallagher P.D., Satija S.K., Phys. Rev. Lett. 72 (1994) 2899.

[18] Leadbetter A.J., Frost, G.C., Gaughan, J.P., Gray, G. W. and Mosley A., J. Phys. France 40 (1979) 375.

[19] Shen Y., Safinya C. R., Fetters L., Adam M., Witten T. and Hadjichristidis N., Phys. Rev. A 43 (1991) 1886.

[20] Safinya C.R., Roux D., Smith G.S., Sinha S.K., Dimon P., Clark N.A., Bellocq A.M., Phys. Rev. Lett. 57 (1986) 2718; Safinya C. R., Sirota E. B., Roux D. and Smith G. S., Phys. Rev. Lett. 62 (1989) 1134; Roux D., Bellocq A.M., in "Physics of amphiphiles: micelles, vesicles and microemulsions", V. Degiorgio and M. Corti Eds. (North Holland, Amsterdam, 1985).

[21] Safinya C. R., in "Phase Transitions in Soft Condensed Matter", T. Riste and D. Sherrington Eds. Nato ASI Series B 211 (1989) 249; Roux D., Safinya C.R., Nallet F., in "Micelles, membranes, microemulsions and monolayers". Gelbart W.M,, Ben-Shaul A.. Roux D. Eds. (Springer Verlag, New York, 1994).

[22] Diat O. and Roux D., J. Phys. II France 3 (1993) 1427.

[23] Israelachvili J. N. and McGuiggan P. M., J. Mater. Res. 5 (1990) 2223.

[24] Pieranski P. and Jerome B., Phys. Rev. A 40 (1989) 317.

[25] Horn R.G.. Israelachvili J.N. and Perez E., J. Phys. France 42 (1981) 39.

[26] Koppi K. A., Tirrell M., Bates F. S., Almdal K. and Colby R. H., J. Phys. France 2 (1992) 1941; Koppi K. A., Tirrell M. and Bates F. S., Phys. Rev. Lett. 70 (1993) 1449.

[27] Safinya C.R., Liang F.S., Varady W.A., Clark N.A. and Andersson G., Phys. Rev. Lett. $\mathbf{5 3}$ (1984) 1172.

[28] Fontes E., Heiney P.A., Ohba M., Haseltine J.N. and Smith A.B. III, Phys. Rev. A 37 (1988) 1329.

[29] Davidson P., Clerc M., Ghosh S.S., Maliszewskyj N.C., Heiney P.A., Hynes Jr J. and Smith III A.B., J. Phys. II France 5 (1995) 249.

[30] Guinier A., "X-ray diffraction" (Freeman, New York, 1963).

[31] Porte G., Marignan J., Bassereau P. and May R., Europhys. Lett. 7 (1988) 713.

[32] Nallet F., Roux D. and Milner S.T., J. Phys. France 51 (1990) 2333.

[33] Lei N., Safinya C.R. and Bruinsma R.F., J. Phys. II France 5 (1995) 1155. 